\title{
Modelo logístico difásico no estudo do crescimento de fêmeas da raça Hereford
}

\author{
Difasics logistic model in the study of the growth of Hereford breed females
}

\author{
Patrícia Neves Mendes ${ }^{\mathrm{I}}$ Joel Augusto Muniz ${ }^{\mathrm{I}^{*}}$ Fabyano Fonseca e Silva $^{\mathrm{II}}$ \\ Ana Rita de Assumpção Mazzini' ${ }^{\text {III }}$
}

\section{RESUMO}

Este trabalho teve como objetivo comparar modelos logísticos difásicos ponderados aplicados ao estudo de curvas de crescimento de fêmeas Hereford com três diferentes estruturas de erros: erros independentes (EI), auto-regressivos de primeira ordem (AR (1)) e auto-regressivo de segunda ordem (AR (2)) a dados de peso-idade de 55 fêmeas da raça Hereford avaliadas desde o nascimento até 675 dias de idade. Utilizouse o procedimento model do software Statistical Analysis System (SAS) por meio das opções weight e \%AR. A comparação entre os modelos foi realizada com base na interpretação biológica dos parâmetros e nos avaliadores de qualidade de ajuste (coeficiente de determinação ajustado, teste de DurbinWatson, desvio padrão residual, número de iterações), além do critério de informação de Akaike (AIC) e do teste F para comparação de modelos. Os resultados obtidos para o ajuste dos modelos aos dados médios indicaram que o modelo logístico difásico AR (2) foi o mais eficiente para descrever a curva de crescimento do rebanho. Ao se considerar o conjunto de dados individuais, nenhum dos modelos abordados foi recomendado por produzirem estimativas não condizentes com a realidade.

Palavras-chave: modelos não-lineares, erros auto-regressivos, heterogeneidade de variâncias.

\section{ABSTRACT}

This study had the objective of comparing weighted difasics logistic models applied to the study of Hereford females growth curves with three different error structures: independent errors (IE), first-order auto-regressive (AR (1)) and secondorder auto-regressive (AR (2)) to weight-age data of 55 females of the Hereford breed, raised in the Bagé region, RS, Brazil, evaluated from birth to 675 days old. The weight and \%AR options of model procedure, available in the software Statistical Analysis System (SAS), was used to fit data. The comparison among the models was carried out through the biological interpretation basis of the parameters and in the adjustment of quality measures (adjusted determination coefficient, DurbinWatson test, residual standard desviation, number of iterations), beyond the Akaike information criteria (AIC) and the F test for model comparison. The models fitted to mean data indicated that the difasic logistic with AR(2) structure was the most efficient to describe the herd growth curve. In the individual fit, none of the models was accepted because they didn't produce consistent estimates.

Key words: non-linear models, auto-regressive errors, heterogenety of variances.

\section{INTRODUÇÃO}

Atualmente, uma forma prática e consistente de analisar a eficiência produtiva de raças de bovinos de corte é por meio do estudo de curvas de crescimento, pois estas representam uma trajetória longitudinal dos pesos apresentados pelos animais em função do tempo. Raças zebuínas, como Nelore e Guzerá, as quais são tradicionalmente criadas em praticamente todas as regiões brasileiras, já tiveram suas curvas de crescimentos reportadas em inúmeros trabalhos na literatura (por exemplo, OLIVEIRA et al. 2000; SILVA et al., 2002). Porém, raças européias, que sempre apresentaram grande potencial produtivo, ainda são carentes de pesquisa em relação à identificação de seus padrões de crescimento.

IDepartamento de Ciências Exatas, Universidade Federal de Lavras (UFLA), 37200-000, Lavras, MG, Brasil. E-mail: joamuniz@ufla.br.*Autor para correspondência.

IDepartamento de Informática, Setor de Estatística, Universidade Federal de Viçosa (UFV), Viçosa, MG, Brasil.

IIIDepartamento de Estatística, Universidade Federal de Pelotas (UFPel), Pelotas, RS, Brasil. 
Entre as raças de gado de corte européias criadas no Brasil, destaca-se a raça Hereford, originária da Inglaterra, que possui algumas vantagens consideráveis, tais como: adaptação aos mais diversos ambientes e sistemas de produção, alto índice de fertilidade, bom ganho de peso a pasto e alto índice de rendimento de carcaça. Segundo a Associação Brasileira de Hereford e Braford (2006), responsável pela seleção e pelo registro dos produtos desta raça, o peso mínimo das novilhas com idade de 2 a 2 anos e meio é de $320 \mathrm{~kg}$ de peso vivo.

No estudo de curvas de crescimento, geralmente, são realizados ajustes de modelos nãolineares a dados de peso-idade dos animais. Esses modelos apresentam parâmetros com significado biológico, o que facilita o entendimento a respeito do processo de crescimento. De acordo com SILVA et al. (2001), os modelos mais utilizados, Gompertz (LAIRD \& HOWARD, 1967), Logístico (LAIRD \& HOWARD, 1967), Bertalanffy (BERTALANFFY, 1957) e Brody (BRODY, 1945), apresentam dois parâmetros de grande importância para a avaliação do crescimento, o peso assintótico e a taxa de maturidade. Tais modelos também contêm parâmetros que se caracterizam como constantes matemáticas, os quais não são zootecnicamente relevantes. O peso assintótico ou o peso adulto representa a estimativa de peso à maturidade, independente de flutuações de pesos em razão de efeitos genéticos e ambientais. A taxa de maturidade corresponde a uma estimativa de precocidade, determinando assim a eficiência do crescimento, pois quanto maior seu valor mais precoce é o animal.

Quando o crescimento apresenta um comportamento característico de forma a identificar ciclos, os quais permitem dividir a curva em várias fases, torna-se necessário adotar modelos de crescimento multifásicos, por contemplarem parâmetros exclusivos para cada fase (KOOPS, 1986). Esta situação é muito comum quando se tem efeitos sazonais, os quais, em estudos de curvas de crescimento de bovinos, podem ser caracterizados pelas épocas seca e chuvosa. A ausência de chuvas, com impacto nas pastagens, restringe o crescimento por algum período de tempo, porém, este volta a seguir sua trajetória com a mudança de estação. Na realidade, o que se faz é considerar, em um único modelo, dois ou mais submodelos, cada um com o objetivo de explicar uma determinada fase do crescimento.

$\mathrm{Na}$ maioria dos estudos de curvas de crescimento, algumas pressuposições estatísticas importantes não são levadas em consideração, tais como a heterogeneidade de variância dos pesos no tempo decorrente do aumento da idade, o que alguns autores definiram como "distúrbios de regressão" e a existência de autocorrelação entre os resíduos do ajuste, tendo em vista que os dados são tomados longitudinalmente em cada animal (MAZZINI et al., 2005). Caso estas estatísticas sejam ignoradas, pode ocorrer a obtenção de estimativas viesadas (PASTERNAK \& SHALEV, 1994) e a subestimação das variâncias dos parâmetros, respectivamente (SOUZA, 1998).

O objetivodesse trabalho foi ajustar a função de crescimento logística difásica ponderada considerando diferentes estruturas de autocorrelação para os resíduos a dados de peso-idade de fêmeas da raça Hereford com o intuito de indicar quais os melhores modelos de acordo com avaliadores estatísticos e a interpretação biológica das estimativas dos parâmetros.

\section{MATERIAL E MÉTODOS}

Os dados utilizados neste trabalho provêm de 55 fêmeas da raça Hereford, nascidas nos anos de 1999 a 2001, na Agropecuária Recreio, situada no município de Bagé, Estado do Rio Grande do Sul, cujos pesos foram tomados de 15 em 15 dias, desde o nascimento até, aproximadamente, 675 dias de idade.

Os modelos utilizados foram o Logístico difásico com estrutura de erros independentes (EI), Logístico difásico com estruturas de erros AR (1) e Logístico difásico com estruturas de erros AR (2), os quais, segundo TORNERO (1996), são dados, respectivamente, por:

$$
\begin{aligned}
& \mathrm{Y}_{\mathrm{t}}=\frac{a_{1}}{\left(1+\exp \left\{-\mathrm{k}_{1} *\left(t-d_{1}\right)\right\}\right)}+\frac{a_{2}}{\left(1+\exp \left\{-\mathrm{k}_{2} *\left(t-d_{2}\right)\right\}\right)}+e_{t} \\
& \mathrm{Y}_{\mathrm{t}}=\frac{a_{1}}{\left(1+\exp \left\{-\mathrm{k}_{1} *\left(t-d_{1}\right)\right\}\right)}+\frac{a_{2}}{\left(1+\exp \left\{-\mathrm{k}_{2} *\left(t-d_{2}\right)\right\}\right)}+e_{t}+\phi_{1} e_{t-1} \\
& \mathrm{Y}_{\mathrm{t}}=\frac{a_{1}}{\left(1+\exp \left\{-\mathrm{k}_{1} *\left(t-d_{1}\right)\right\}\right)}+\frac{a_{2}}{\left(1+\exp \left\{-\mathrm{k}_{2} *\left(t-d_{2}\right)\right\}\right)}+e_{t}+\phi_{1} e_{t-1}+\phi_{2} e_{t-2}
\end{aligned}
$$

em que: $Y_{t}$ é o peso corporal, em kg; $t$ é o tempo, em dias; $a_{1}$ e $a_{2}$ são os pesos máximos (estabilização do crescimento), respectivamente para as fases 1 e $2 ; k_{1} \mathrm{e}$ $k_{2}$ são as taxas de maturidade (velocidade de crescimento ou precocidade), respectivamente para as fases 1 e 2 ; $d_{1}$ e $d_{2}$ são as abscissas do ponto de inflexão da curva (não apresenta significado biológico), respectivamente para as fases 1 e 2; e é o resíduo no tempo t; $e_{t-1}$ é o resíduo no tempo t-1; $e_{t-2}$ é o resíduo no tempo t-2, e os parâmetros $\phi_{1}$ e $\phi_{2}$ correspondem, respectivamente, aos parâmetros de auto-regressão residual de primeira e segunda ordem. De acordo com TORNERO (1996), o peso assintótico (A) ou o peso 
adulto do animal é dado pela soma dos pesos máximos de cada fase, ou seja, $A=a_{1}+a_{2}$, porém esta relação não é valida para os outros parâmetros do modelo.

Os modelos não-lineares apresentados não possuem uma solução explícita para o sistema de equações normais responsáveis pela estimação dos parâmetros do modelo, a qual, desse modo, deve ser obtida por meio de processos iterativos, usando algum software estatístico.

Os ajustes foram realizados pelo procedimento model do SAS ${ }^{\circledR}$ (1995), utilizando duas estruturas de dados, dados médios e individuais. $\mathrm{Na}$ primeira, os valores da variável dependente dizem respeito às médias dos pesos dos animais em cada data de avaliação e, na segunda, cada indivíduo apresenta o seu conjunto particular de observações, o que permite avaliar a qualidade dos modelos diante de variações de peso referentes a cada animal. Utilizou-se este procedimento (proc model) devido à possibilidade de se ajustar erros auto-regressivos por meio da macro \%AR(y,p), o que não é permitido no procedimento $n l i n$, geralmente usado em estudos de curvas de crescimento. A ponderação dos dados foi feita pela opção weight, utilizando-se o inverso das variâncias dos pesos também calculadas em cada pesagem. As estimativas iniciais, necessárias para a obtenção das estimativas dos parâmetros por meio do processo iterativo, foram obtidas de MAZZINI et al. (2005). Foram considerados os seguintes avaliadores de qualidade de ajuste: coeficiente de determinação ajustado $\left(\mathrm{R}^{2}{ }_{\mathrm{a}}\right)$, desvio padrão residual (DPR), teste de Durbin-Watson (DW), número de iterações, critério de informação de Akaike (AIC) e o teste F para a comparação de modelos.

As expressões dos avaliadores, segundo MOTULSKY \& CHRISTOPOULOS (2003), são dadas

$$
\begin{aligned}
& R_{a}^{2}=1-\left[\frac{(n-1)\left(1-R^{2}\right)}{(n-p)}\right], D P R=\sqrt{\frac{Q M E}{(n-p)},} \\
& D W=\frac{\sum_{t=2}^{n}\left(e_{t}-e_{t-1}\right)^{2}}{\sum_{t=1}^{n} e_{t}^{2}}, A I C=n * \ln \left(\sigma_{k, l}^{2}\right)+2 * p, \\
& F=\frac{\left(S Q R_{(\text {menor })}-S Q R_{(\text {maior })}\right) /\left(g l_{(\text {menor })}-g l_{(\text {maior })}\right)}{\left(S Q R_{(\text {maior })} / g l_{(\text {maior })}\right)},
\end{aligned}
$$

em que: $\mathrm{R}^{2}=1-(\mathrm{SQR} / \mathrm{SQT})$, SQR é a soma de quadrados dos resíduos, SQT é a soma de quadrados total, QME é o quadrado médio residual, n é o número de observações, $p$ é o número de parâmetros no modelo, $\sigma_{\mathrm{k}, \mathrm{l}}^{2}$ é a variância dos resíduos, $\mathrm{SQR}_{(\text {menor) }}$ é a soma de quadrados de resíduos do modelo com menor número de parâmetros; $\mathrm{SQR}_{\text {(maior) }}$ é a soma de quadrados de resíduos do modelo com mais parâmetros; $\mathrm{gl}_{\text {(menor) }}$ é o grau de liberdade do modelo com menor número de parâmetros; $\mathrm{gl}_{\text {(maior) }}$ é o grau de liberdade do modelo com maior número de parâmetros.

\section{RESULTADOS E DISCUSSÃO}

Os avaliadores da qualidade de ajuste para os dados médios (Tabela 1) indicaram que o modelo logístico difásico AR (2) apresentou maior $\mathrm{R}^{2}$, embora sua vantagem em relação aos demais seja irrisória, e menor DPR. Além disso, o modelo em questão não apresentou significância para a autocorrelação de primeira ordem, caracterizando-se assim como o único a respeitar a pressuposição de independência residual. Em contrapartida, o ajuste deste modelo exigiu maior número de iterações para a convergência, fato que pode ser explicado pela sua maior complexidade. Quanto ao critério de informação de Akaike (AIC), que compara os modelos levando em conta o número de parâmetros envolvidos, observou-se que o modelo logístico difásico AR (2) mesmo sendo penalizado por apresentar um maior número de parâmetros, foi aquele que mostrou maior qualidade de ajuste. Este modelo também foi considerado o melhor pelo teste $\mathrm{F}$, o qual rejeitou a hipótese nula de que o modelo mais simples está correto, o que pode ser verificado pela significância do teste.

Os resultados apresentados na tabela 1 estão de acordo com SILVA et al. (2002) e MAZZINI et al. (2005), os quais também analisaram dados médios de crescimento de bovinos de corte e observaram uma maior qualidade de ajuste para modelos que incorporaram parâmetros auto-regressivos. No entanto, esses autores relataram que tais conclusões não podem ser expandidas para dados individuais.

Na tabela 2 são mostrados os percentuais de convergência e as médias dos QME e dos $\mathrm{R}^{2}$, ou seja, estes dois últimos avaliadores apresentam-se como resultados médios provenientes dos ajustes individuais dos modelos estudados. Nota-se por esta tabela que a inclusão de estrutura de erros autoregressivos dificultou a convergência do modelo, resultado que está de acordo com o encontrado por MAZZINI et al. (2003) no estudo de machos Hereford.

Ao serem realizdos ajustes individuais podem surgir determinadas variações nos pesos, fato este não observado nos ajustes médios. Estas correspondem, muitas vezes, a observações discrepantes ocasionadas pela avaliação de animais cujos pesos estão muito acima ou muito abaixo da média do rebanho e também pela ocorrência de erros na 
Tabela 1 - Avaliadores da qualidade do ajuste considerados: número de parâmetros (№ parms.), coeficiente de determinação ajustado ( $\mathrm{R}^{2}{ }_{\mathrm{a}}$ ), desvio padrão residual (DPR), teste de Durbin-Watson (DW), número de iterações (№ iter.), critério de informação de Akaike (AIC) e teste F, para curva média.

\begin{tabular}{lcccccc}
\hline Modelos & No parms. & $\mathrm{R}_{\mathrm{a}}^{2}$ & DPR & DW & No iter. & AIC \\
\hline Logístico Difásico & 6 & 0,9851 & 0,6128 & $0,2452^{*}$ & 18 & $-37,483$ \\
Logístico Difásico AR (1) & 7 & 0,9973 & 0,2621 & $0,8347^{*}$ & 30 & $-114,76$ \\
Logístico Difásico AR (2) & 8 & 0,9992 & 0,1466 & 1,9311 & 35 & $-167,45$ \\
\hline
\end{tabular}

* Significativo a $5 \%$ de probabilidade.

** Significativo a $1 \%$ de probabilidade.

anotação dos dados. Ao se trabalhar com dados médios, estas variações são diluídas e observa-se uma maior suavização na curva de crescimento dos animais, o que resulta, na maioria das vezes, em maiores facilidades de convergência (SILVA et al., 2002). Segundo SOUZA (1998), ao aumentar a complexidade do modelo, por exemplo, ao serem utilizadas estruturas de erros auto-regressivos, espera-se uma maior dificuldade de convergência, e este fato aliado com as variações individuais já relatadas, explicam a baixa porcentagem de convergência do modelo Logístico difásico AR (2). Assim, este modelo não é recomendado para a análise de curvas de crescimento individuais, pois apenas $30,90 \%$ dos animais puderam ter seus parâmetros de crescimento estimados, e tal afirmação discorda dos resultados obtidos para os dados médios.

De acordo com o exposto, o modelo com estrutura de erros AR (1) apresenta boas características para descrever as curvas de crescimento individuais, pois o mesmo apresenta-se em uma situação intermediária, mostrando boa qualidade de ajuste mediante os critérios QME e $\mathrm{R}^{2}$, a qual é melhor que aquela apresentada pela estrutura de erros independentes (EI), e uma porcentagem de convergência relativamente alta, 81,81\%, e não tão distante dos $100 \%$ observado para a estrutura EI.

Observa-se ainda na tabela 2, que a inclusão de estruturas de erros mais complexas ocasiona uma

Tabela 2 - Avaliadores da qualidade para o ajuste das curvas individuais - Percentual de convergência (\% convergência), quadrado médio do erro (QME) e coeficiente de determinação ajustado $\left(\mathrm{R}^{2}{ }_{\mathrm{a}}\right)$.

\begin{tabular}{lccc}
\hline \multirow{2}{*}{ Avaliador } & \multicolumn{3}{c}{------Estrutura de erros------- } \\
& EI $^{*}$ & AR (1) & AR (2) \\
\hline \% Convergência & 100,00 & 81,81 & 30,90 \\
QME & 181,68 & 84,84 & 77,84 \\
$\mathrm{R}^{2} \mathrm{a}$ & 0,9582 & 0,9806 & 0,9819 \\
\hline
\end{tabular}

${ }^{*} \mathrm{EI}=$ erros independentes. diminuição do QME e um aumento do $\mathrm{R}^{2}$, resultados estes que estão de acordo com MAZZINI et al. (2003), que também analisaram curvas de crescimento individuais de gado Hereford.

Em análise de curvas de crescimento, não só o aspecto estatístico deve ser levado em consideração na identificação dos melhores modelos, mas também é necessário avaliar sob o ponto de vista biológico as estimativas dos parâmetros produzidas por eles. Na tabela 3 são mostradas estas estimativas para os ajustes médios e individuais, sendo que neste último os valores mostrados correspondem às médias das estimativas obtidas para os animais cuja convergência foi constatada.

De acordo com os resultados apresentados na tabela 3, nota-se pelas estimativas dos parâmetros $\mathrm{a}_{1}$ e $\mathrm{a}_{2}$ na análise dos dados médios que o modelo com estrutura AR (2) apresentou um valor para o peso adulto (A) mais condizente com a realidade, sendo este de 471,9833 $\left(A=a_{1}+a\right)$, enquanto que para o modelo com estrutura EI e ${ }^{2} A R$ (1) os valores foram respectivamente, 259,7000 e 208,0594. Esta discussão leva em consideração os valores estabelecidos pela Associação Brasileira de Criadores de Hereford e Braford (2006), que exige um peso mínimo de $320 \mathrm{~kg}$ de peso vivo. Segundo GOONEWARDENE et al. (1981), os quais estudaram o crescimento de fêmeas da raça Hereford e seus cruzamentos, e MAZZINI et al. (2005), que avaliaram o crescimento de machos Hereford, o modelo logístico apresenta a característica de superestimar o peso ao nascer e subestimar os pesos finais dos animais, porém, suas estimativas tendem a ser mais realísticas quando as dependências residuais são modeladas. Este fato foi observado no presente trabalho, pois, como já mencionado na discussão da tabela 1, a estrutura AR (2) foi aquela que descreveu de forma mais eficiente a autocorrelação residual.

Ainda em relação à interpretação biológica dos parâmetros, tendo em vista o conjunto de dados médios, observa-se na tabela 3 que ao se considerar as estruturas EI e AR (1) a taxa de maturidade da primeira fase $\left(k_{1}\right)$ foi menor que a da segunda $\left(k_{2}\right)$, indicando 
Tabela 3 - Estimativas dos parâmetros e erros padrões (entre parênteses) provenientes dos ajustes médios e individuais, para as três estruturas de erros consideradas: Erros independentes, Auto-regressivos de ordem 1 (AR (1)) e Auto-regressivos de ordem 2 (AR (2)).

\begin{tabular}{|c|c|c|c|}
\hline Estrutura de erros & Parâmetros* & Ajustes médios ${ }^{1}$ & Ajustes individuais ${ }^{2}$ \\
\hline & $\mathrm{a}_{1}$ & $144,2000(3,7238)$ & $143,8130(2,385)$ \\
\hline & $\mathrm{k}_{1}$ & $0,0188(0,0012)$ & $0,0210(0,0003)$ \\
\hline \multirow{7}{*}{ Independentes } & $\mathrm{d}_{1}$ & $70,7585(4,5130)$ & $71,2000(1,2949)$ \\
\hline & $\mathrm{a}_{2}$ & $115,5000(8,2012)$ & $114,8920(2,7388)$ \\
\hline & $\mathrm{k}_{2}$ & $0,0365(0,0089)$ & $0,0380(0,0017)$ \\
\hline & $\mathrm{d}_{2}$ & $479,6000(8,2121)$ & $480,7510(3,1348)$ \\
\hline & $a_{1}$ & $139,4000(8,3156)$ & $138,4000(2,7400)$ \\
\hline & $\mathrm{k}_{1}$ & $0,0196(0,0015)$ & $0,0200(0,0053)$ \\
\hline & $\mathrm{d}_{1}$ & $69,0544(7,6664)$ & $72,2600(1,5800)$ \\
\hline \multirow{7}{*}{$\mathrm{AR}(1)$} & $\mathrm{a}_{2}$ & $68,6594(22,6227)$ & $87,7684(28,3253)$ \\
\hline & $\mathrm{k}_{2}$ & $0,0536(0,0195)$ & $0,1000(0,0400)$ \\
\hline & $\mathrm{d}_{2}$ & $454,4000(9,1567)$ & $643,4000(73,8700)$ \\
\hline & $\phi_{1}$ & $1,1611(0,0825)$ & $0,9200(0,0500)$ \\
\hline & $\mathrm{a}_{1}$ & $61,5833(5,8910)$ & $137,2200(2,8100)$ \\
\hline & $\mathrm{k}_{1}$ & $0,0444(0,0042)$ & $0,0200(0,0071)$ \\
\hline & $\mathrm{d}_{1}$ & $3,9882(3,9574)$ & $68,9700(1,4600)$ \\
\hline \multirow{5}{*}{$\operatorname{AR}(2)$} & $\mathrm{a}_{2}$ & $410,4000(114,8)$ & $122,8111(28,6559)$ \\
\hline & $\mathrm{k}_{2}$ & $0,0231(0,0041)$ & $0,0300(0,0141)$ \\
\hline & $\mathrm{d}_{2}$ & $458,8000(14,3103)$ & $403,4600(15,0400)$ \\
\hline & $\phi_{1}$ & $2,0599(0,0442)$ & $1,0000(0,0500)$ \\
\hline & $\phi_{2}$ & $-1,0804(0,0445)$ & $-0,2600(0,0400)$ \\
\hline
\end{tabular}

* åe a - pesos máximos (estabilização do crescimento), respectivamente para as fases 1 e 2;

$k_{1}$ e $k_{2}$ - taxas de maturidade (velocidade de crescimento), respectivamente para as fases 1 e 2 ;

$d_{1}$ e $d_{2}$ - abscissas do ponto de inflexão da curva (não apresenta significado biológico), respectivamente para as fases 1 e 2;

e - resíduo no tempo t; e-1 - resíduo no tempo t-1; e-2 - resíduo no tempo t-2;

$\phi_{1}$ e $\phi_{2}$ correspondem, respectivamente, aos parâmetros de auto-regressão residual de primeira e segunda ordem.

${ }^{1}$ médias dos pesos dos animais em cada data de avaliação;

${ }^{2}$ média dos ajustes aos pesos individuais em todas as datas de avaliação.

que o animal cresce mais rapidamente nesta última. De acordo com TORNERO (1996), isso não é verdade, pois se espera que a velocidade de crescimento dos animais seja maior na primeira fase, de forma a se observar uma diminuição em sua magnitude de acordo com a utilização de novas fases. Dessa forma, a estrutura de erros AR (2) também se caracteriza como a mais concordante com a realidade, pois proporcionou os valores $0,0444 \mathrm{e}$ 0,0231 respectivamente para os parâmetros $\mathrm{k}_{1}$ e $\mathrm{k}_{2}$.

Ainda em relação à tabela 3 , porém focando os resultados obtidos na análise individual, pode-se notar que o peso adulto dos animais foi subestimado por todos os modelos considerados e que a taxa de precocidade para o modelo AR (2) não manteve o mesmo comportamento decrescente observado na análise dos dados médios. Sendo assim, os modelos utilizados não são indicados, sob o ponto de vista biológico, para descrever curvas de crescimento individuais, as quais são de grande interesse para área de Melhoramento Genético Animal. Porém, ao serem considerados os dados médios, análise a qual é de grande importância para a otimização de manejo e a avaliação da rentabilidade do rebanho, o modelo AR (2) é recomendado por proporcionar alta qualidade de ajuste e estimativas de acordo com o padrão racial.

Na figura 1(a), é possível visualizar o comportamento dos resíduos ao longo do tempo considerando o ajuste dos dados médios. De acordo com o gráfico, percebe-se claramente que eles não estão dispostos aleatoriamente em torno do zero, apresentando um comportamento cíclico, o qual evidencia a presença de autocorrelação residual. Considerando o aspecto prático, é razoável aceitar que os erros tendem a acompanhar a variação do ganho de peso dos animais, isto é, em épocas chuvosas em que há mais disponibilidade de forragens, os animais “ganham” mais peso; em contrapartida, na época seca, 

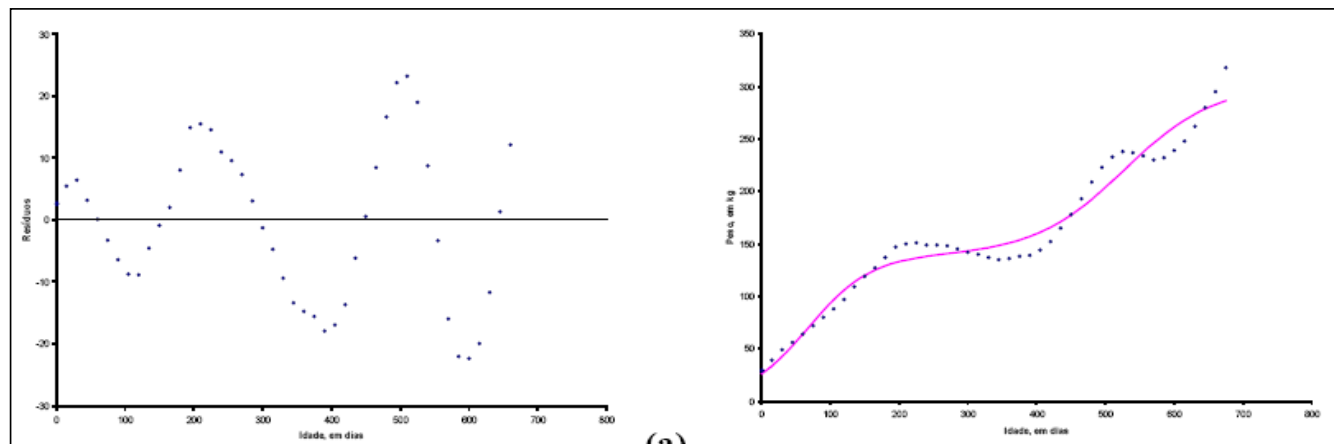

(a)
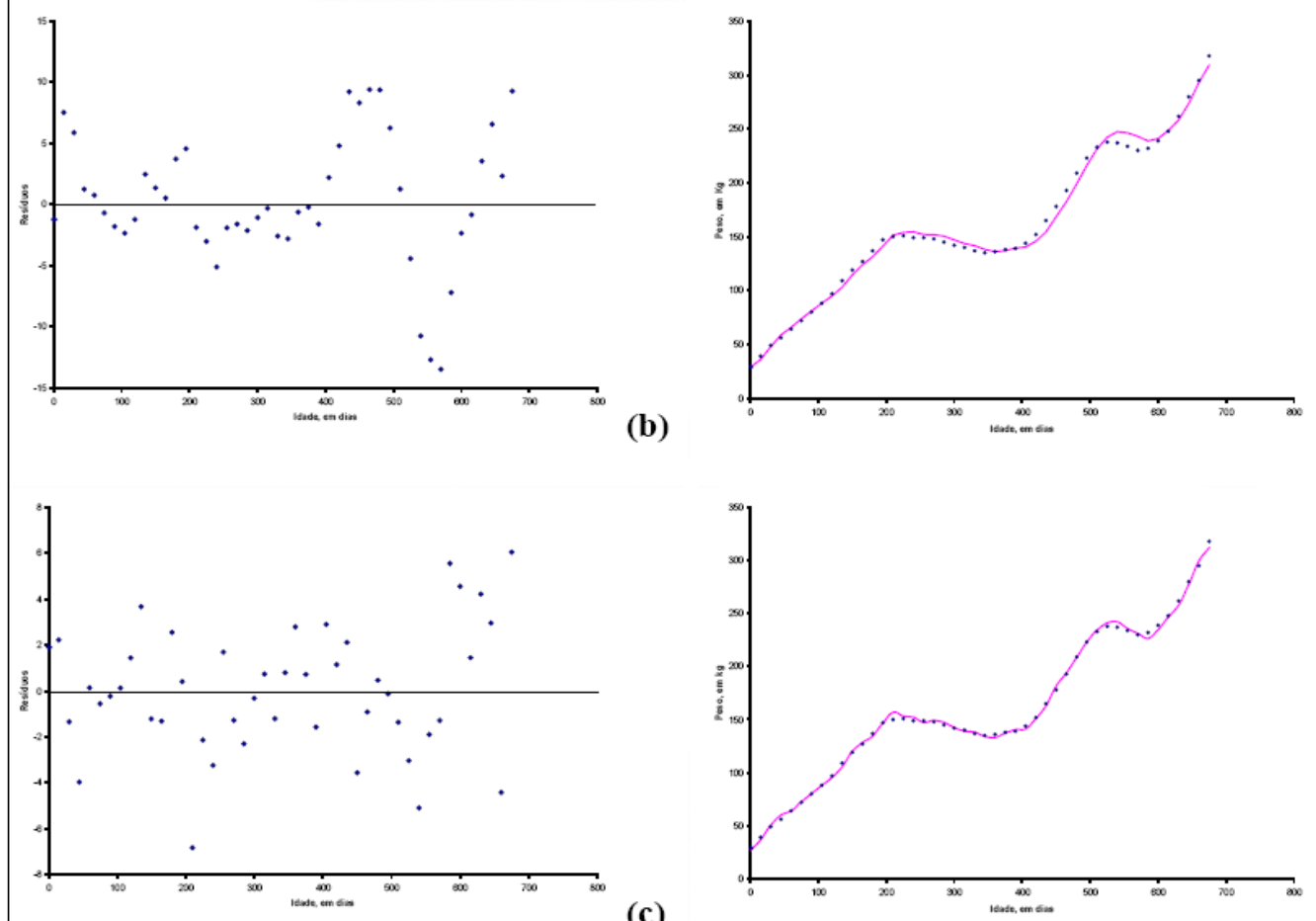

Figura 1 - Resíduos do modelo logístico difásico e ajuste da função logística difásica com estrutura de erros EI* (a), AR (1) (b) e AR (2) (c).

${ }^{*} \mathrm{EI}=$ erros independentes.

quando há escassez de alimento e o frio é intenso por causa da região, os animais tendem a "perder" peso. Tal comportamento se repete o ano todo de acordo com as estações, e o mesmo já foi relatado por TORNERO (1996), a que estudou o crescimento de vacas holandesas criadas a pasto.

Ainda em relação aos resíduos, percebe-se, nas figuras 1(b) e 1(c), diferentemente da figura 1(a), que existe uma tendência dos mesmos em se mostrarem aleatoriamente distribuídos em torno do zero, sendo esta tendência mais evidente na figura 1(c). Dessa forma, entende-se que realmente é necessário buscar a modelagem da dependência residual, pois a introdução da estrutura de erros auto-regressivos de ordem 2 praticamente solucionou o problema da dependência residual, visto que o comportamento aleatório foi obtido. Este fato também foi comprovado pelo teste de Durbin-Watson (Tabela 1), o qual revelou-se não significativo. Resultados semelhantes foram verificados por MEDEIROS (2000), que, ao estudar o crescimento de vacas holandesas, contornou o problema da autocorrelação quando levou em consideração o modelo com estrutura de erros AR (1).

Na mesma figura 1 (gráficos à direita), podese verificar o ajuste da função logística aos dados observados, sendo possível notar que ao serem adotadas as estruturas de erros auto-regressivos a 
qualidade do ajuste realmente melhora, sendo esta melhora evidente com a estrutura AR (2).

\section{CONCLUSÃO}

De acordo com os resultados obtidos para o ajuste dos modelos aos dados médios, pode-se concluir que o logístico difásico AR (2) apresentou boa qualidade em relação aos aspectos estatísticos e biológicos, sendo então recomendado para descrever a curva de crescimento do rebanho em questão.

O modelo AR (1) apresentou boa qualidade de ajuste ao se considerar o conjunto de dados individuais, porém, não produziu estimativas para os parâmetros condizentes com a realidade, o que leva a não recomendação de nenhum dos modelos abordados para estudos de curvas de crescimento individuais.

\section{REFERÊNCIAS}

ASSOCIAÇÃO BRASILEIRA DE HEREFORD \& BRAFORD. Hereford - carne de qualidade tipo exportação. Capturado em 10 set. 2006. Online. Disponível na Internet: http://www.hereford.com.br/?bW9kdWxvPTEmbW VudT01NiZhcnF1aXZvPWNvbnRldWRvLnBocA.

BERTALANFFY, L. Quantitative laws in metabolism ang growth. Quarterly Review Biology, Chicago, v.32, p.217, 1957.

BRODY, S. Bioenergetics and growth. New York: Reinhold, 1945.

GOONEWARDENE, L.A. et al. A growth study of beef cattle. Canadian Journal of Animal Science, Ottawa, v.61, n.4, p.1041-1048, 1981

KOOPS, W.J. Multiphasic growth curve analysis. Growth 50, Bar Harbor, n.2, p.169-177, 1986.

LAIRD, A.K.; HOWARD, A. Growth curves in inbred mice. Nature, London, v.213, n.5078, p.786-788, 1967.

MAZZINI, A.R.A. et al. Análise da curva de crescimento de machos Hereford. Ciência e Agrotecnologia, Lavras, v.27, n.5, p.1105-1112, 2003.
MAZZINI, A.R.A. et al. Curvas de crescimento de novilhos Hereford: heterocedasticidade e resíduos autorregressivos. Ciência Rural, Santa Maria, v.35, n.2, p.422-427, 2005.

MEDEIROS, H.A. et al. Avaliação da qualidade do ajuste da função logística monofásica com estrutura de erros independentes e autorregressivos através de simulação. Ciência e Agrotecnologia, Lavras, v.24, n.4, p.973-985, 2000.

MOTULSKY, H.; CHRISTOPOULOS, A. Fitting models to biological data using linear and nonlinear regression: a practical guide to curve fitting. 4.ed. San Diego, CA: GraphPad Software, 2003. 351p.

OLIVEIRA, H.N. de. et al. Comparação de modelos não-lineares para descrever o crescimento de fêmeas da Raça Guzerá. Pesquisa Agropecuária Brasileira, Brasília, v.35, n.9, p.1843-1851, 2000.

PASTERNAK, H.; SHALEV, B.A. The effect of a feature of regression disturbance on the efficiency of fitting growth curves. Growth, Development \& Aging, Bar Harbor, v.58, n.1, p.3339, 1994.

SAS INSTITUTE. SAS/ETS User's guide. Version 6. 2.ed. Cary, 1995.

SILVA, F.F. et al. Estimativas de parâmetros genéticos de curva de crescimento de gado Nelore (Bos indicus). Ciência e Agrotecnologia, Lavras, v.26, p.1562-1567, 2002. (Edição Especial).

SILVA, F.F. et al. Influência de fatores genéticos e ambientais sobre as estimativas dos parâmetros das funções de crescimento em gado nelore. Ciência e Agrotecnologia, Lavras, v.25, n.5, p.1195-1205, 2001.

SOUZA, G. da S. Introdução aos modelos de regressão linear e não-linear. Brasília: Embrapa-SPI/Embrapa - SEA, 1998. 489p.

TORNERO, M.T.T. Utilização de modelos assintóticos sigmóides de crescimento multifásico com sazonalidade em estudos zootécnicos. 1996. 131f. Tese (Doutorado em Zootecnia/ Nutrição e Produção Animal) - Faculdade de Medicina Veterinária e Zootecnia da Universidade Estadual Paulista, Botucatu. 